\title{
EP-89
}

\section{Efficacy of entecavir verses lamivudine prophylaxis in preventing hepatitis $B$ virus reactivation in patients with allogeneic stem cell transplant}

\author{
Dexton JOHNS*
}

Clinical Research, National Medicity Hospital, India

Introduction: Immunosuppressive therapy has been the main stay of treatment in patients with hematological malignancies. Reactivation of occult hepatitis B infection (HBV), is commonly encountered with stem cell transplant recipients with anti CD20 agents, with history of resolved HBV infection. We aimed to find the efficacy of nucleos(t)ide analogues lamivudine (LVD) and entecavir (ETV) in preventing the HBV flare up when given prophylactically.

Methods: Patients who received allogeneic stem cell transplant were evaluated. Sixty patients, thirty in each group who received LVD or ETV prophylaxis were included in the study. All the baseline and post-transplant data was evaluated which includes anti -CD20 agent exposure, duration of prophylaxis of each drugs and clinical and virological follow up post transplantation. Serum ALT, HBsAg, anti HBs and HBV DNA were the parameters measured.

Results: The median clinical and virological follow up in each group was 24 months. LVD or ETV was given during the entire follow up period. There was no reactivation during the prophylaxis period, however 2 patients in LVD group showed detectable viral DNA post prophylaxis withdrawal.

Conclusions: The clinical outcome of both LVD and ETV reveals equal efficacy in preventing HBV reactivation. However screening is mandatory before chemotherapy. HBs Ag-negative/anti-HBc-positive patients require ALT monitoring during the entire chemotherapy. 